\title{
Tendencias en el Uso de Redes Sociales Para Educación
}

\section{Fernando J. Garrigós Simón, Sofía Estellés Miguel, Ismael Lengua Lengua, Jose Onofre Montesa Andrés, Juan Vicente Oltra Gutierreza , Yeamduan Narangajavana Kaosiri ${ }^{\mathbf{b}}$.}

aUniversitat Politècnica de València, Valencia, Spain, fgarrigos@doe.upv.es, soesmi@omp.upv.es, ilengua@dig.upv.es, jomontes@omp.upv.es, jvoltra@omp.upv.es, ${ }^{\mathrm{b}}$ Universitat Jaume I, Castellón, Spain, ynaranga@uji.es.

\begin{abstract}
Resumen
El uso de las redes socials en educación es muy relevante, tanto para educadores como estudiantes, para promover el aprendizaje. Ello ha sido observado por la literatura, la cual observa un importante crecimiento en el número de artículos analizando dicho tema. Sin embargo este estudio es todavía escaso. El objetivo de este artículo es presentar una revisión bilométrica del uso de las redes sociales en educación, con objeto de identificar el desarrollo del área, y sus tendencias. Para ello, el trabajo utiliza datos de la Web of Science, además de instrumenos bibliométricos y el programa VOS viewer, enfatizando el estudio de la co-ocurrencia de palabra clave. Dado ello, el articulo subraya las tendencias actuales en el area, hecho que puede ayudar a investigadores y profesionales para entender el estado del arte en el área.
\end{abstract}

Palabras clave: Educación, Redes sociales, Bibliométrico, Visualización, tendencias.

\section{Introducción}

El análisis de las redes socials en la literature en general y específicamente en la literatura de educación ha experimentado un crecimiento importante en los útimos años, convirtiéndose en un tópico de investigación esencial en esta área. Dado ello, el propósito de este artículo es el desarrollar un análisis bibliométrico de la literatura que engloba simultáneamente ambas áreas de educación y social media (ESM).

En la literatura todavía hay una comprensión limitada del significado de "Social Media", siendo elusiva todavía su definición (Kaplan y Haenlein, 2010). Sin embargo, nosotros podríamos definir las redes sociales como sistemas tecnológicos (aplicaciones móviles 
basadas en la Web) las cuales permiten a organizaciones e indiviuos comunicas y colaborar con otros usuarios de forma virtual, a través de crear y compartin contenido o información existente o creado explícitamente po los propios usuarios. En este sentido, las redes sociales permiten en general, o específicamente en nuestro contexto a educadores y estudiantes el poder tomar ventaje del uso de información relevante para sus propósitos. La relevancia de las redes sociales es ccrucial. Así, el desarrollo de tecnologías de información Internet, y la impotante e imprecedente difusión de plataformas digitales tales como Facebook, Twitter, Youtube, Wikipedia, o Instagram en las últimas décadas, ha implicado una rápida e incluso ubicua adopción de las redes sociales a través de muy divestas esperas de la sociedad (Sugimoto, Work, Larivière, y Haustein, 2017). Obviamente, este desarrollo en la esfera social, se ha trasladado de forma rápida a la academica, con un incremento muy relevante y continuo del número de trabajos que analizan tan fenómeno.

El ámbito educativo no ha sido una excepción de este hecho. Ello es especialmente relevante est este campo, segun Dabbagh y Kitsantas (2012), debido a la existendia de una evidencia importante de que las redes sociales pueden facilitar la creación de entornos personales de aprendizaje. Además, las redes sociales puede proveer a los estudiantes con un rol más activo en el desarrollo de su propia educación, pudendo observarse tanto como productores como consumidores de la misma (Martín et al., 2011). En este sentido, numerosos autores consideran la integración de las redes socials como un hecho importante como herramienta educative, existiendo por ello una amplio interés en la investigación en aplicar herramientas relacionadas con las redes sociales en la educación contemporánea (Piotrowski, 2015).

No obstante, aun cuanto el estudio de las redes sociales, junto a la aplicación actual de dispositivos móviles, representan dos de las áreas de desarrollo más importantes relativas al uso de tecnologías de educación en el futuro próximo, su estudio todavía necesita de investigación intensiva, existiendo en particular poco conocimiento sobre la extensión de la infestigación en redes sociales en la literatura (Piotrowski, 2015).

Una forma de abordar esta cuestión es a través de la utilización de análisis bibliométricos. En la literatura existen muy diversos análisis bibliométricos acerca de la relevancia de las redes sociales en general, o aplicadas a diversas áreas. También existen diversos bibliométricos aplicados al área de educación, e incluso un estudio bibliométrico reciente que estudia el uso de algunas redes sociales como Facebook en la investigación educativa (Lopes, Fidalgo-Neto, y Mota, 2017). Sin embargo, existe una falta de investigación sobre el uso de las redes sociales en educación en general. Con objeto de afrontar esta carencia, el objetivo de este trabajo es realizar un análisis bibliométrico sobre la evolución de ESM 


\section{Metodología}

Tal y como hemos expuesto, este trabajo se centra en un análisis bibliométricos Esta es una metodología que analiza de forma cuantitativa el material bibliográfico, con métodos matemáticos y estadísticos (Broadus, 1987), siendo actualmenta ampliamente utilizada para identificiar el desarrollod de campos en diverrsa áreas, pudiéndose establecer a partir del mismo importantes conclusiones (Garrigós et al., 2018). Además, el trabajo utiliza el análisis grafico para visulaizar los resultados, a través del programa VOS viewer software. A su vez, realizamos un análisis de co-ocurrencia de palabras clave de autor (palabras clave de los autores que aparecen juntas debajo de los abstracs) para poder estudiar los tópicos más importantes en la disciplina.

La investigación se basa en los resultados encontrados en la base de datos de la "Web of Science Corre Collection" (WoS), siguiendo los consejos de diversos autores que aconsejan su uso frente a otras como Scopus o Google Scholar, por sus altos estándares académicos (Delgado López-Cózar, Robinson-García, y Torres-Salinas, 2014; Garrigos et al., 2018).

Para seleccionar la muestra utilizamos las palabras clave "Social Media" y "educación” . La población considera todos los trabajos publicados hasta el 31 de diciembre de 2017. La población fue reducida considerando solo artículos, revisiones, cartas y notas, con objetivo de focalizarnos en los trabajos principales, obteniendo una muestra final de 1962 documentos.

\section{Resultados}

Aun y cuando existen artículos relacionados con las redes sociales en la WoS en 1997, el incremento en las publicacones sobre esta temática comenzó después de 2005, sobrepasando los 100 artículos anuales en 2010 y llegando a más de 4000 en 2017. Esta evolución fue paralela en el área de educación, donde los primeros trabajos aparecieron en 2009, sobrepasando ya los 500 anuales en 2017.

No obstante, este trabajo quiere incidir fundamentalmente en las principales palabras clave, para observar la evolución del área, realizando un análisis de coocurrencia de las palabras calve de autor, como explicamos previamente. En este sentido en tre las 1962 publicaciones observadas relacionadas con ESM, encontramos 4191 palabras clave. La figura I muestra las palabras y el tamaño de los nodos (a mayor tamaño de palabra y nodo, mayor peso tiene; a menor distancia entre los nodos mayor la relación entre las palabras, a mayor grosor de la línea, mayor coocurrencia). Los nodos con el mismo color pertenecen a un mismo clúster. En este sentido el programa creo 12 cluster. La figura considera un umbral de 5 ocurrencias, mostrando loas 208 mayores co-ocurrencias. VOS viewer divide las palabras 
clave. Aparte de "Social Media", las palabras clave más importantes son "Facebook", "Education", "Twitter" y "Higher Education"

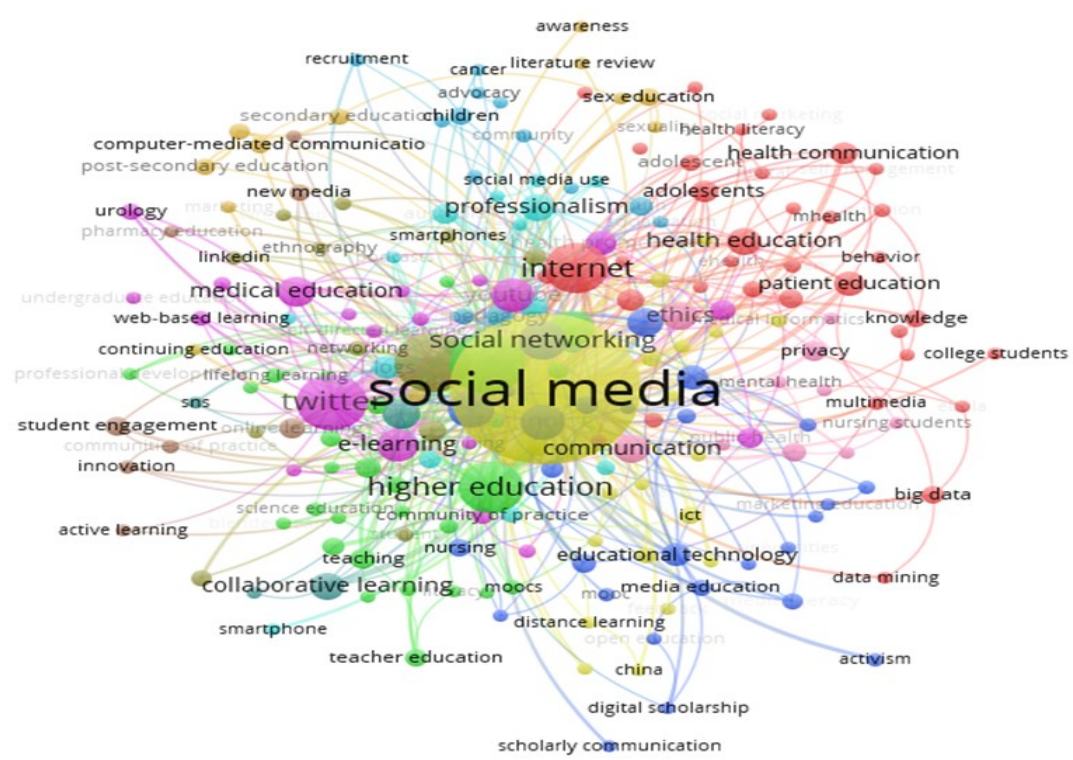

Fig. 1. Red de Co-ocurencia de las palabras clave de autor de las publicaciones relacionadas con ESM

\section{Discusión y Conclusiones}

La parte teória de ste trabajo ha definido a las redes sociales en general, ha analizado su importancia en el campo educativo, y se ha concentrado en la importancia de poder realizar un bibliométirco para el análisis de la evolución de la literatura sobre resdes sociales en educación. A su vez, el trabajo ha realizado un análisis bibliométrico, con análisis de cocourrencia de las palabras clave, a partir del cual se pueden extraer diversas conclusiones. El análisis observa que además de "Social Media", las palabra clave más importante son " "Facebook", "Education", "Twitter" and "Higher Education". Es relevante a este respecto la centralidad de la palabra Facebook, y su conexión con términos relevantes y centrales como "Education", "technology", "web 2.0", "social networking" o "networking". Debemos señalar también la relevancia de términos asociados con medicina, y el uso importante de internet y las redes sociales por parte de este campo. A este respecto observamos la inclusión en el mismo cluster de la palabra "Twitter" "e-learning", "youtube", "medical education" "health promotion" y "urology"; la inclusión de "health 
education" o "health communication" o "patient education" en el cluster liderado por el termino "internet", que también incluye "knowledge"o "big data". Creemos relevante también el surgimiento de clusters, todavía poco relevantes, pero que creemos que tienen potencial, liderados respectivamente por los términos "'collaborative learning", "educational technology", o "professionalism".

Nuestro trabajo creo que ha producido resultados interesantes. A este respecto abre la puerta al estudio de las áreas más relevantes o incipientes, ofreciendo un marco importante del estado del arte de la cuestión. Sin embargo, el trabajo tiene numerosas limitaciones, que abren la puerta a posteriores trabajos. Además de obviar otros importantes estudios bibliométricos (co-citas, co-autoría..., o estudios de análisis de trabajos particulares, autores, instituciones, o países), el trabajo podría completarse con la atención a otras bases de datos, o el empleo de software complementario. .

\section{Referencias}

Broadus, R. (1987). Toward a definition of "bibliometrics". Scientometrics, 12(5-6), 373-379.

Dabbagh, N., y Kitsantas, A. (2012). Personal Learning Environments, social media, and selfregulated learning: A natural formula for connecting formal and informal learning. The Internet and higher education, 15(1), 3-8.

Delgado López-Cózar, Emilio, Nicolás Robinson-García, y Daniel Torres-Salinas (2014). "The Google Scholar experiment: How to index false papers and manipulate bibliometric indicators." Journal of the Association for Information Science and Technology 65(3), 446-454.

Garrigos-Simon, F., Narangajavana-Kaosiri, Y., y Lengua-Lengua, I. (2018). Tourism and Sustainability: A Bibliometric and Visualization Analysis. Sustainability, 10(6), 1976.

Kaplan, A. M., y Haenlein, M. (2010). Users of the world, unite! The challenges and opportunities of Social Media. Business horizons, 53(1), 59-68.

Lopes, R. M., Fidalgo-Neto, A. A., y Mota, F. B. (2017). Facebook in educational research: a bibliometric analysis. Scientometrics, 111(3), 1591-1621.

Martin, S., Diaz, G., Sancristobal, E., Gil, R., Castro, M., y Peire, J. (2011). New technology trends in education: Seven years of forecasts and convergence. Computers \& Education, 57(3), 1893-1906

Piotrowski, C. (2015). Emerging Research on Social Media Use in Education: A Study of Dissertations. Research in Higher Education Journal, 27.

Sugimoto, C. R., Work, S., Larivière, V., y Haustein, S. (2017). Scholarly use of social media and altmetrics: A review of the literature. Journal of the Association for Information Science and Technology, 68(9), 2037-2062 